K. J. Falconer, Mathematical Institute, University of St Andrews, North Haugh, St Andrews, Fife, KY16 9SS, UK. e-mail: kjf@st-and.ac.uk M. Järvenpää and P. Mattila, University of Jyväskylä, Department of Mathematics, P.O. Box 35, FIN-40351 Jyväskylä, Finland. e-mail: amj@math.jyu.fi and pmattila@math.jyu.fi

\title{
EXAMPLES ILLUSTRATING THE INSTABILITY OF PACKING DIMENSIONS OF SECTIONS
}

\begin{abstract}
We shall use the "iterated Venetian blind" construction to show that the packing dimensions of plane sections of subsets of $\mathbb{R}^{n}$ can depend essentially on the directions of the planes. We shall also establish the instability of the packing dimension of sections under smooth diffeomorphisms.
\end{abstract}

\section{Introduction and Notation}

Let $m$ and $n$ be integers with $0<m<n$. We use the notation $\gamma_{n, m}$ for the unique orthogonally invariant Radon probability measure on the Grassmann manifold $G_{n, m}$ consisting of all $m$-dimensional linear subspaces of $\mathbb{R}^{n}$. The uniqueness of $\gamma_{n, m}$ implies that there is a positive and finite constant $c$ depending on $m$ and $n$ such that for all $A \subset G_{n, m}$

$$
\begin{aligned}
& \gamma_{n, m}(A)=c\left(\mathcal{H}^{n} \times \cdots \times \mathcal{H}^{n}\right)\left(\left\{\left(y_{1}, \ldots, y_{m}\right) \in\left(\mathbb{R}^{n}\right)^{m}:\left|y_{i}\right| \leq 1\right.\right. \\
&\text { for all } \left.\left.i=1, \ldots, m \text { and } V\left(y_{1}, \ldots, y_{m}\right) \in A\right\}\right)
\end{aligned}
$$

where $\mathcal{H}^{n}$ is $n$-dimensional Hausdorff measure and $V\left(y_{1}, \ldots, y_{m}\right)$ is the $m$ dimensional linear subspace spanned by the vectors $y_{1}, \ldots, y_{m}$. For $V \in G_{n, m}$ we denote by $\operatorname{proj}_{V}$ the orthogonal projection onto $V$, by $V^{\perp}$ the orthogonal complement of $V$, and by $V_{a}$ the $m$-plane $\{v+a: v \in V\}$ for $a \in V^{\perp}$.

Key Words: packing dimension, plane sections, iterated constructions

Mathematical Reviews subject classification: 28A12, 28A80

Received by the editors April 15, 1999 
For Borel sets $E \subset \mathbb{R}^{n}$ one has the following very precise information about the Hausdorff dimension, $\operatorname{dim}_{\mathrm{H}}$ (for the definition see [F2, Chapter 2] or [Mat3, Chapter 4], of projections and plane sections of $E$ (see [K], [Mar], and [Mat1]): for $\gamma_{n, m}$-almost all $V \in G_{n, m}$

$$
\operatorname{dim}_{\mathrm{H}} \operatorname{proj}_{V}(E)=\min \left\{m, \operatorname{dim}_{\mathrm{H}} E\right\}
$$

and

$$
\mathcal{H}^{n-m}\left(\left\{a \in V^{\perp}: \operatorname{dim}_{\mathrm{H}}\left(E \cap V_{a}\right)=\operatorname{dim}_{\mathrm{H}} E-(n-m)\right\}\right)>0
$$

provided that in (1.3) $\operatorname{dim}_{\mathrm{H}} E \geq n-m$ and $0<\mathcal{H}^{\operatorname{dim}_{\mathrm{H}} E}(E)<\infty$.

Note that for the Hausdorff and packing dimensions, $\operatorname{dim}_{\mathrm{H}}$ and $\operatorname{dim}_{\mathrm{p}}$, (for the definition see [F2, Chapter 3] or [Mat3, Chapter 5], of sections we have the following natural upper bounds: if $E \subset \mathbb{R}^{n}$ and $V \in G_{n, m}$, then

$$
\operatorname{dim}_{\mathrm{H}}\left(E \cap V_{a}\right) \leq \max \left\{0, \operatorname{dim}_{\mathrm{H}} E-(n-m)\right\}
$$

and

$$
\operatorname{dim}_{\mathrm{p}}\left(E \cap V_{a}\right) \leq \max \left\{0, \operatorname{dim}_{\mathrm{p}} E-(n-m)\right\}
$$

for $\mathcal{H}^{n-m}$-almost all $a \in V^{\perp}$ (see [F3, Lemma 5] and [Mat 3, Chapter 10]). For the packing dimension, the formulae (1.2) and (1.3) are false, but there are weaker results for both sets and measures (see [FH1], [FH2], [FJ], [FM], and $[\mathrm{JM}]$ ). Although there is no formula such as (1.2) for the packing dimensions of projections, Falconer and Howroyd showed in [FH2] that given an analytic set $E \subset \mathbb{R}^{n}, \operatorname{dim}_{\mathrm{p}} \operatorname{proj}_{V}(E)$ is almost surely a constant; that is, there is a number $d_{m}(E)$ such that $\operatorname{dim}_{\mathrm{p}} \operatorname{proj}_{V}(E)=d_{m}(E)$ for $\gamma_{n, m}$-almost all $V \in G_{n, m}$. The purpose of this paper is to show that there is no such result for plane sections. We shall prove that there exists a compact set $E \subset \mathbb{R}^{n}$ and compact subsets $A$ and $B$ of $G_{n, m}$ with $\gamma_{n, m}(A)>0$ and $\gamma_{n, m}(B)>0$ such that for all $V \in A$ we have $\mathcal{H}^{m}\left(\operatorname{proj}_{V^{\perp}}(E)\right)=0$; that is, $E \cap V_{a}=\emptyset$ for $\mathcal{H}^{n-m}$-almost all $a \in V^{\perp}$, and for all $V \in B$ we have $\operatorname{dim}_{\mathrm{p}}\left(E \cap V_{a}\right)=m$ for points $a$ in a non-empty open subset of $V^{\perp}$. Quite likely, but perhaps with considerable technical complications, it would be possible to show that given a Borel function $f$ from the space of affine $m$-planes in $\mathbb{R}^{n}$ into the closed interval $[0, m]$ there is a Borel set $E \subset \mathbb{R}^{n}$ such that $\operatorname{dim}_{\mathrm{p}}(E \cap V)=f(V)$ for almost all affine $m$-planes $V$. This would be analogous to the results of Davies [D] and Falconer [F1] where $A_{V} \subset V$ is given in an arbitrary but measurable way and then $E$ is found such that for $\gamma_{n, m}$-almost all $V \in G_{n, m} \operatorname{proj}_{V}(E)$ agrees with $A_{V}$ up to a set of $m$-dimensional measure zero. (After completing this paper we learned that Csörnyei $[\mathrm{C}]$ has extended our results by proving this conjecture in the plane.) 
In Section 5 we shall establish the instability of the packing dimensions of sections under smooth "bending" diffeomorphisms. We shall show that given a $C^{2}$-diffeomorphism $f: A \rightarrow B$ between two plane domains $A$ and $B$ which does not map every line segment onto a line segment there is a compact subset $E$ of $A$ such that $\mathcal{H}^{1}\left(\operatorname{proj}_{L}(E)\right)=0$ for $\gamma_{2,1}$-almost all $L \in G_{2,1}$; that is, almost all sections $E \cap L_{a}$ are empty, but for all $L \in G_{2,1}$ we have $\operatorname{dim}_{\mathrm{p}}\left(f(E) \cap L_{a}\right)=1$ for all points $a$ in some non-empty open subset of $L^{\perp}$.

\section{The Basic Result for Hyperplanes in $\mathbb{R}^{n}$}

In this section we begin a two-stage induction process that proves the result on which our first construction is based. Here we consider hyperplanes in $\mathbb{R}^{n}$ and in the next section we work with general $m$-planes in $\mathbb{R}^{n}$.

Let $P \subset[0,1]^{n}$ be a non-degenerate closed parallelepiped. We name the edges of $P$ such that the shortest parallel edges are called 1-edges, the second shortest parallel edges are 2-edges and so on. This numbering distinguishes edges which are not parallel; that is, if two edges have the same length but they are not parallel, then they have different numbers. For all $i=1, \ldots, n$ we call $P_{i}^{1}$ and $P_{i}^{2}$ the $(n-1)$-faces of $P$ which are generated by the edges numbered by $1, \ldots, i-1, i+1, \ldots, n$.

For our purposes it is enough to consider a specific class of subparallelepipeds of $[0,1]^{n}$. Let $\left\{x_{1}, \ldots, x_{n}\right\}$ be the standard basis of $\mathbb{R}^{n}$. For all $i=$ $1, \ldots, n$ we let $W_{i}$ be the hyperplane spanned by $\left\{x_{1}, \ldots, x_{i-1}, x_{i+1}, \ldots, x_{n}\right\}$. We call $P \subset[0,1]^{n}$ a hyperregular parallelepiped in $\mathbb{R}^{n}$ if $P_{i}^{1}$ and $P_{i}^{2}$ are parallel to $W_{i}$ for all $i \neq n-1$; let $P_{i}^{1}$ be the one that is nearest to $W_{i}$. For a hyperregular parallelepiped $P$ and $\delta>0$ we define

$$
\begin{array}{r}
\mathcal{A}_{n, n-1}(P)=\{V: V \text { is an affine }(n-1) \text {-plane meeting both } \\
\left.P_{i}^{1} \text { and } P_{i}^{2} \text { for all } i \neq n \text { but not } P_{n}^{1} \text { and } P_{n}^{2}\right\}
\end{array}
$$

and

$$
\mathcal{A}_{n, n-1}^{\delta}(P)=\left\{V \in \mathcal{A}_{n, n-1}(P): \operatorname{dist}\left(V \cap P, P_{n}^{2}\right) \geq \delta\right\}
$$

where $\operatorname{dist}\left(V \cap P, P_{n}^{2}\right)=\inf \left\{|a-b|: a \in V \cap P, b \in P_{n}^{2}\right\}$ is the distance between $V \cap P$ and $P_{n}^{2}$.

The following lemma from [Mat2] describes the plane case underlying the basic construction for hyperplanes in higher dimensions.

Lemma 2.1. There are disjoint compact sets $A, B \subset G_{2,1}$ with $\gamma_{2,1}(A)>0$ and $\gamma_{2,1}(B)>0$ such that for all hyperregular parallelograms $P \subset[0,1]^{2}$ and 
for all $\varepsilon>0$ there exists a finite family $\mathcal{P}_{\varepsilon}$ of hyperregular subparallelograms of $P$ with the following properties:

1. $\mathcal{H}^{1}\left(\operatorname{proj}_{L^{\perp}}\left(\cup \mathcal{P}_{\varepsilon}\right)\right) \leq \varepsilon$ for all $L \in A$.

2. There is $\delta>0$ such that if $L \in \mathcal{A}_{2,1}(P) \cap \mathcal{A}_{2,1}\left([0,1]^{2}\right)$ is parallel to some line belonging to $B$, then there exists $Q \in \mathcal{P}_{\varepsilon}$ such that $L \in \mathcal{A}_{2,1}^{\delta}(Q)$.

Proof. See [Mat2, Lemma 2]. Note that in the plane we can parametrize the lines through the origin by the angle they make with the positive $x_{1}$ axis. Using this parametrization [Mat2, Lemma 2] gives $A=[a, a+b]$ and $B=[0, a-b] \cup[a+2 b, \pi]$ where $a$ and $b$ are real numbers with $0<b<a$ and $a+2 b<\pi$.

Next we prove the higher-dimensional version of Lemma 2.1 for hyperplanes.

Lemma 2.2. There are disjoint compact sets $A, B \subset G_{n, n-1}$ with $\gamma_{n, n-1}(A)>$ 0 and $\gamma_{n, n-1}(B)>0$ such that for all hyperregular parallelepipeds $P \subset[0,1]^{n}$ and for all $\varepsilon>0$ there exists a finite family $\mathcal{P}_{\varepsilon}$ of hyperregular subparallelepipeds of $P$ with the following properties:

1. $\mathcal{H}^{1}\left(\operatorname{proj}_{V^{\perp}}\left(\cup \mathcal{P}_{\varepsilon}\right)\right) \leq \varepsilon$ for all $V \in A$.

2. There is $\delta>0$ such that if $V \in \mathcal{A}_{n, n-1}(P) \cap \mathcal{A}_{n, n-1}\left([0,1]^{n}\right)$ is parallel to some hyperplane belonging to $B$, then there exists $Q \in \mathcal{P}_{\varepsilon}$ such that $V \in \mathcal{A}_{n, n-1}^{\delta}(Q)$.

Proof. If $n=2$, the result is a restatement of Lemma 2.1. We assume inductively that the claim holds in $\mathbb{R}^{n-1}$ and deduce the result in $\mathbb{R}^{n}$.

We may restrict our consideration to hyperregular parallelepipeds $P$ with $P_{1}^{1} \subset W_{1}$. We use the notation $\gamma_{W_{1}, n-2}$ for the invariant measure on the Grassmann manifold $G_{W_{1}, n-2}$ of all $(n-2)$-dimensional linear subspaces of $W_{1}$. Applying the induction hypothesis to $W_{1}$ which is identified with $\mathbb{R}^{n-1}$ and defining $\mathcal{A}_{W_{1}, n-2}(\widetilde{P})$ and $\mathcal{A}_{W_{1}, n-2}^{\delta}(\widetilde{P})$ in the obvious way for hyperregular parallelepipeds $\widetilde{P} \subset[0,1]^{n-1}$ in $W_{1}$ and for $\delta>0$, we find disjoint compact sets $\widetilde{A}, \widetilde{B} \subset G_{W_{1}, n-2}$ with $\gamma_{W_{1}, n-2}(\widetilde{A})>0$ and $\gamma_{W_{1}, n-2}(\widetilde{B})>0$ such that for all hyperregular parallelepipeds $\widetilde{P} \subset[0,1]^{n-1}$ and for all $\varepsilon>0$ there exists a finite family $\widetilde{\mathcal{P}}_{\varepsilon}$ of hyperregular subparallelepipeds of $\widetilde{P}$ such that

$$
\mathcal{H}^{1}\left(\operatorname{proj}_{V^{\perp, W_{1}}}\left(\cup \widetilde{\mathcal{P}}_{\varepsilon}\right)\right) \leq \varepsilon
$$

for all $V \in \widetilde{A}$. Here $\operatorname{proj}_{V^{\perp, W_{1}}}: W_{1} \rightarrow V^{\perp, W_{1}}$ is the orthogonal projection onto the orthogonal complement $V^{\perp, W_{1}} \in G_{W_{1}, 1}$ of $V$. Further, there is $\delta>0$ such 
that if $V \in \mathcal{A}_{W_{1}, n-2}(\widetilde{P}) \cap \mathcal{A}_{W_{1}, n-2}\left([0,1]^{n-1}\right)$ is parallel to some $(n-2)$-plane belonging to $\widetilde{B}$, then

$$
V \in \mathcal{A}_{W_{1}, n-2}^{\delta}(\widetilde{Q})
$$

for some $\widetilde{Q} \in \widetilde{\mathcal{P}}_{\varepsilon}$.

Define

$$
A=\left\{V \in G_{n, n-1}: V \cap W_{1} \in \widetilde{A}\right\}
$$

and

$$
B=\left\{V \in G_{n, n-1}: V \cap W_{1} \in \widetilde{B}, 0 \leq \operatorname{angle}\left(x_{1}, V \cap\left(V \cap W_{1}\right)^{\perp}\right) \leq \pi / 4\right\},
$$

where angle $\left(x_{1}, V \cap\left(V \cap W_{1}\right)^{\perp}\right)$ is the angle between the $x_{1}$-axis and the line $V \cap\left(V \cap W_{1}\right)^{\perp}$ measured on $\left(V \cap W_{1}\right)^{\perp} \in G_{n, 2}$. Here the positivity of the angle is determined by requiring that the half-line

$$
V \cap\left(V \cap W_{1}\right)^{\perp} \cap\left\{\left(y_{1}, \ldots, y_{n}\right) \in \mathbb{R}^{n}: y_{1} \geq 0\right\}
$$

intersects the $(n-1)$-plane where $x_{n}=1$. In this way we fix the positive direction of the angle for all $\left(V \cap W_{1}\right)^{\perp} \in G_{n, 2}$ which are not subsets of $W_{n}$. For the rest of the 2-planes $\left(V \cap W_{1}\right)^{\perp}$ we do this in some fixed sense; it turns out that either of the two possibilities will do.

Clearly $A$ and $B$ are disjoint. Since $\gamma_{W_{1}, n-2}(\widetilde{A})>0$ and $\gamma_{W_{1}, n-2}(\widetilde{B})>0$, it is easy to see from (1.1) that $\gamma_{n, n-1}(A)>0$ and $\gamma_{n, n-1}(B)>0$.

Let $P \subset[0,1]^{n}$ be a hyperregular parallelepiped with $P_{1}^{1} \subset W_{1}$ and let $\varepsilon>0$. Since $\widetilde{P}=P \cap W_{1}$ is a hyperregular parallelepiped in $W_{1}$, there exists by the induction hypothesis a finite family $\widetilde{\mathcal{P}}_{\varepsilon}$ of hyperregular subparallelepipeds of $\widetilde{P}$ such that (2.1) and (2.2) hold. Let $V \in A$. Since

$$
\operatorname{proj}_{V^{\perp}}\left(\cup \widetilde{\mathcal{P}}_{\varepsilon}\right)=\operatorname{proj}_{V^{\perp}} \operatorname{proj}_{\left(V \cap W_{1}\right)^{\perp}}\left(\cup \widetilde{\mathcal{P}}_{\varepsilon}\right)=\operatorname{proj}_{V^{\perp}} \operatorname{proj}_{\left(V \cap W_{1}\right)^{\perp, W_{1}}}\left(\cup \widetilde{\mathcal{P}}_{\varepsilon}\right)
$$

we obtain from (2.1) that

$$
\mathcal{H}^{1}\left(\operatorname{proj}_{V^{\perp}}\left(\cup \widetilde{\mathcal{P}}_{\varepsilon}\right)\right) \leq \varepsilon .
$$

Let $\mathcal{P}_{\varepsilon}$ be a finite family of hyperregular subparallelepipeds of $P$ obtained by extending the parallelepipeds of $\widetilde{\mathcal{P}}_{\varepsilon}$ to very thin parallelepipeds to the direction of the positive $x_{1}$-axis. Then (1) holds by $(2.3)$.

Let $\delta>0$ be as in (2.2). If $V \in \mathcal{A}_{n, n-1}(P) \cap \mathcal{A}_{n, n-1}\left([0,1]^{n}\right)$ is parallel to some hyperplane belonging to $B$, then $V \cap W_{1} \in \mathcal{A}_{W_{1}, n-2}(\widetilde{P}) \cap$ $\mathcal{A}_{W_{1}, n-2}\left([0,1]^{n-1}\right)$ is parallel to some $(n-2)$-plane belonging to $\widetilde{B}$. Using (2.2), we find $\widetilde{Q} \in \widetilde{\mathcal{P}}_{\varepsilon}$ such that $V \cap W_{1} \in \mathcal{A}_{W_{1}, n-2}^{\delta}(\widetilde{Q})$. Since $0 \leq \operatorname{angle}\left(x_{1}, V \cap\right.$ 
$\left.\left(V \cap W_{1}\right)^{\perp}\right) \leq \pi / 4$ and since we may choose the length of the 1-edges of the parallelepipeds of $\mathcal{P}_{\varepsilon}$ to be less than $\delta / 2$, we have $V \in \mathcal{A}_{n, n-1}^{\delta / 2}(Q)$ where $Q \in \mathcal{P}_{\varepsilon}$ is the enlargement of $\widetilde{Q}$. Note that since here $V \in \mathcal{A}_{n, n-1}\left([0,1]^{n}\right)$ is parallel to some $V_{p} \in B$, the $x_{n}$-axis cannot be a subset of $V_{p} \cap W_{1}$. Thus $\left(V_{p} \cap W_{1}\right)^{\perp}$ is not a subset of $W_{n}$. In this case the positiveness of angle $\left(x_{1}, V_{p} \cap\left(V_{p} \cap W_{1}\right)^{\perp}\right)$ is explicitly defined.

\section{The Extension of the Basic Result to $m$-Planes in $\mathbb{R}^{n}$}

In order to extend the result of Lemma 2.2 for general $m$-planes in $\mathbb{R}^{n}$ we do a two-stage induction process: first we use the results of the previous section for hyperplanes and then we prove the general case. As before we restrict our attention to a certain class of parallelepipeds. We say that a non-degenerate closed parallelepiped $P \subset[0,1]^{n}$ is an m-regular parallelepiped in $\mathbb{R}^{n}$ if $P$ is of the form $S \times[0,1]^{n-(m+1)}$ where $S \subset[0,1]^{m+1}$ is a hyperregular parallelepiped in $\mathbb{R}^{m+1}$. We number the edges of $P$ in the same way as before and define for all $i=1, \ldots, n$ the $(n-1)$-faces $P_{i}^{1}$ and $P_{i}^{2}$ as before. Note that for all $i \neq m$ both $P_{i}^{1}$ and $P_{i}^{2}$ are parallel to $W_{i}$. For an $m$-regular parallelepiped $P \subset[0,1]^{n}$ we set

$$
\begin{aligned}
\mathcal{A}_{n, m}(P)= & \left\{V: V \text { is an affine } m \text {-plane meeting both } P_{i}^{1} \text { and } P_{i}^{2}\right. \\
& \text { for all } \left.i=1, \ldots, m \text { but not } P_{i}^{1} \text { and } P_{i}^{2} \text { when } i=m+1, \ldots, n\right\} .
\end{aligned}
$$

Lemma 3.1. There are disjoint compact sets $A, B \subset G_{n, m}$ with $\gamma_{n, m}(A)>0$ and $\gamma_{n, m}(B)>0$ such that for all $m$-regular parallelepipeds $P \subset[0,1]^{n}$ and for all $\varepsilon>0$ there exists a finite family $\mathcal{P}_{\varepsilon}$ of $m$-regular subparallelepipeds of $P$ with the following properties:

1. $\mathcal{H}^{n-m}\left(\operatorname{proj}_{V^{\perp}}\left(\cup \mathcal{P}_{\varepsilon}\right)\right) \leq \varepsilon$ for all $V \in A$.

2. If $V \in \mathcal{A}_{n, m}(P) \cap \mathcal{A}_{n, m}\left([0,1]^{n}\right)$ is parallel to some $m$-plane belonging to $B$, then there exists $Q \in \mathcal{P}_{\varepsilon}$ such that $V \in \mathcal{A}_{n, m}(Q)$.

Proof. If $n=m+1$, the result is a consequence of Lemma 2.2. Keeping $m$ fixed, we assume inductively that the result holds in $\mathbb{R}^{n-1}$ and prove it in $\mathbb{R}^{n}$.

Identifying $W_{n}$ with $\underset{\widetilde{A}}{\mathbb{R}^{n-1}}$ and using the induction hypothesis, we find disjoint compact sets $\widetilde{A}, \widetilde{B} \subset G_{W_{n}, m}$ with $\gamma_{W_{n}, m}(\widetilde{A})>0$ and $\gamma_{W_{n}, m}(\widetilde{B})>0$ such that for all $m$-regular parallelepipeds $\widetilde{P} \subset[0,1]^{n-1}$ and for all $\varepsilon>0$ there exists a finite family $\widetilde{\mathcal{P}}_{\varepsilon}$ of $m$-regular subparallelepipeds of $\widetilde{P}$ such that for all $V \in \widetilde{A}$

$$
\mathcal{H}^{n-1-m}\left(\operatorname{proj}_{V^{\perp, W_{n}}}\left(\cup \widetilde{\mathcal{P}}_{\varepsilon}\right)\right) \leq \varepsilon .
$$


Further, if $V \in \mathcal{A}_{W_{n}, m}(\widetilde{P}) \cap \mathcal{A}_{W_{n}, m}\left([0,1]^{n-1}\right)$ is parallel to some $m$-plane belonging to $\widetilde{B}$, then

$$
V \in \mathcal{A}_{W_{n}, m}(\widetilde{Q})
$$

for some $\widetilde{Q} \in \widetilde{\mathcal{P}}_{\varepsilon}$.

Define

$$
A=\left\{V \in G_{n, m}: \operatorname{proj}_{W_{n}}(V) \in \widetilde{A}\right\}
$$

and

$$
B=\left\{V \in G_{n, m}: \operatorname{proj}_{W_{n}}(V) \in \widetilde{B}\right\} .
$$

Clearly $A$ and $B$ are disjoint compact sets with $\gamma_{n, m}(A)>0$ and $\gamma_{n, m}(B)>0$.

Let $P \subset[0,1]^{n}$ be an $m$-regular parallelepiped and let $\varepsilon>0$. Using the induction hypothesis for the $m$-regular parallelepiped $\widetilde{P}=P \cap W_{n}$ in $W_{n}$ we find a finite family $\left\{\widetilde{P}_{\varepsilon}^{1}, \ldots, \widetilde{P}_{\varepsilon}^{k}\right\}$ of $m$-regular subparallelepipeds of $\widetilde{P}$ such that (3.1) and (3.2) hold. Now $\mathcal{P}_{\varepsilon}=\left\{\widetilde{P}_{\varepsilon}^{1} \times[0,1], \ldots, \widetilde{P}_{\varepsilon}^{k} \times[0,1]\right\}$ is a finite family of $m$-regular subparallelepipeds of $P$. Consider $V \in A$. Note that for $W=\operatorname{proj}_{W_{n}}(V) \in \widetilde{A}$ we have $W^{\perp, W_{n}} \subset V^{\perp}$. Since $\mathcal{H}^{n-m}\left(\operatorname{proj}_{V^{\perp}}\left(\cup \mathcal{P}_{\varepsilon}\right)\right)$ $\leq 2 n \mathcal{H}^{n-1-m}\left(\operatorname{proj}_{W^{\perp, W_{n}}}\left(\cup \mathcal{P}_{\varepsilon}\right)\right)$ and $\operatorname{proj}_{W^{\perp, W_{n}}}\left(\cup \mathcal{P}_{\varepsilon}\right)=\operatorname{proj}_{W^{\perp, W_{n}}}\left(\cup \widetilde{\mathcal{P}}_{\varepsilon}\right)$, we obtain (1) from (3.1). Finally, if $V \in \mathcal{A}_{n, m}(P) \cap \mathcal{A}_{n, m}\left([0,1]^{n}\right)$ is parallel to some $m$-plane belonging to $B$, then for all $i=1, \ldots, n-1$ we have $\operatorname{proj}_{W_{n}}\left(V \cap P_{i}^{j}\right)=\operatorname{proj}_{W_{n}}(V) \cap \widetilde{P}_{i}^{j}$ for $j=1,2$. Since $\operatorname{proj}_{W_{n}}(V) \in \mathcal{A}_{W_{n}, m}(\widetilde{P}) \cap$ $\mathcal{A}_{W_{n}, m}\left([0,1]^{n-1}\right)$ is parallel to some $m$-plane belonging to $\widetilde{B}$, we obtain by (3.2) that $\operatorname{proj}_{W_{n}}(V) \in \mathcal{A}_{W_{n}, m}\left(\widetilde{P}_{\varepsilon}^{l}\right)$ for some $1 \leq l \leq k$ giving $V \in \mathcal{A}_{n, m}\left(\widetilde{P}_{\varepsilon}^{l} \times\right.$ $[0,1])$.

\section{The Main Construction}

Using Lemma 3.1 we prove our main result:

Theorem 4.1. There exist compact sets $E \subset \mathbb{R}^{n}$ and $A, B \subset G_{n, m}$ with $\gamma_{n, m}(A)>0$ and $\gamma_{n, m}(B)>0$ such that

1. for all $V \in A$ we have $\mathcal{H}^{n-m}\left(\operatorname{proj}_{V^{\perp}}(E)\right)=0$, and

2. for all $V \in B$ there exists a non-empty open subset $U_{V}$ of $V^{\perp}$ such that $\operatorname{dim}_{\mathrm{p}}\left(E \cap V_{a}\right)=m$ for all $a \in U_{V}$.

Proof. Let $A, B \subset G_{n, m}$ be as in Lemma 3.1. Setting $P_{1,1}=[0,1]^{n}$ and using Lemma 3.1 we find $m$-regular parallelepipeds $Q_{2,1}, \ldots, Q_{2, l_{2}} \subset P_{1,1}$ such that for all $V \in A$

$$
\mathcal{H}^{n-m}\left(\operatorname{proj}_{V^{\perp}}\left(\bigcup_{q=1}^{l_{2}} Q_{2, q}\right)\right) \leq \frac{1}{2} .
$$


Further, if $V \in \mathcal{A}_{n, m}\left(P_{1,1}\right)$ is parallel to some $m$-plane belonging to $B$, then $V \in \mathcal{A}_{n, m}\left(Q_{2, q}\right)$ for some $1 \leq q \leq l_{2}$. For all $1 \leq q \leq l_{2}$ and $1 \leq i \leq m$ let $e_{i}\left(Q_{2, q}\right)$ be the length of the $i$-edges of $Q_{2, q}$. Let $k_{2}$ be the smallest positive integer such that for all $1 \leq q \leq l_{2}$

$$
k_{2} \geq e_{1}\left(Q_{2, q}\right)^{-2 m+1} .
$$

Dividing each $Q_{2, q}$ into $\left(k_{2}\right)^{m} m$-regular parallelepipeds with all the edges parallel to the corresponding edges of $Q_{2, q}$ and with the length of the $i$-edges equal to $\frac{1}{k_{2}} e_{i}\left(Q_{2, q}\right)$ for all $1 \leq i \leq m$, we obtain $m$-regular parallelepipeds $P_{2,1}, \ldots, P_{2, N_{2}}$ where $N_{2}=l_{2}\left(k_{2}\right)^{m}$. Clearly

$$
\mathcal{H}^{n-m}\left(\operatorname{proj}_{V^{\perp}}\left(\bigcup_{q=1}^{N_{2}} P_{2, q}\right)\right) \leq \frac{1}{2}
$$

for all $V \in A$. By Lemma 3.1 we find for all $1 \leq q \leq N_{2} m$-regular parallelepipeds $Q_{3,1}^{q}, \ldots, Q_{3, l_{3}^{q}}^{q} \subset P_{2, q}$ such that for all $V \in A$

$$
\mathcal{H}^{n-m}\left(\operatorname{proj}_{V^{\perp}}\left(\bigcup_{p=1}^{l_{3}^{q}} Q_{3, p}^{q}\right)\right) \leq \frac{1}{3 N_{2}} .
$$

Further, whenever $V \in \mathcal{A}_{n, m}\left(P_{2, q}\right) \cap \mathcal{A}_{n, m}\left(P_{1,1}\right)$ is an $m$-plane parallel to some $m$-plane belonging to $B$, then $V \in \mathcal{A}_{n, m}\left(Q_{3, p}^{q}\right)$ for some $1 \leq p \leq l_{3}^{q}$. As before, divide each $Q_{3, p}^{q}$ into $\left(k_{3}\right)^{m} m$-regular parallelepipeds with all edges parallel to the corresponding edges of $Q_{3, p}^{q}$ and with the length of the $i$-edges equal to $\frac{1}{k_{3}} e_{i}\left(Q_{3, p}^{q}\right)$ for all $1 \leq i \leq m$. Here $k_{3}$ is the smallest integer such that for all $1 \leq q \leq N_{2}$ and $1 \leq p \leq l_{3}^{q}$ we have $k_{3} \geq e_{1}\left(Q_{3, p}^{q}\right)^{-3 m+1}$. This gives us $m$-regular parallelepipeds $P_{3,1}, \ldots, P_{3, N_{3}}$ where $N_{3}=\sum_{q=1}^{N_{2}} l_{3}^{q}\left(k_{3}\right)^{m}$. Since

$$
\bigcup_{q=1}^{N_{3}} P_{3, q} \subset \bigcup_{q=1}^{N_{2}} \bigcup_{p=1}^{l_{3}^{q}} Q_{3, p}^{q},
$$

by (4.1) we have $\mathcal{H}^{n-m}\left(\operatorname{proj}_{V^{\perp}}\left(\bigcup_{q=1}^{N_{3}} P_{3, q}\right)\right) \leq \frac{1}{3}$.

Continue in this way and define a compact set $E=\bigcap_{p=1}^{\infty} \bigcup_{q=1}^{N_{p}} P_{p, q}$. If $V \in A$, then for all positive integers $p$

$$
\mathcal{H}^{n-m}\left(\operatorname{proj}_{V^{\perp}}(E)\right) \leq \mathcal{H}^{n-m}\left(\operatorname{proj}_{V^{\perp}}\left(\bigcup_{q=1}^{N_{p}} P_{p, q}\right)\right) \leq \frac{1}{p}
$$


giving the first claim.

Finally, let $V \in \mathcal{A}_{n, m}\left(P_{1,1}\right)$ be parallel to some $m$-plane belonging to $B$. By the construction for all $j$ we have $V \in \mathcal{A}_{n, m}\left(Q_{j, p}^{q}\right)$ for some $1 \leq q \leq N_{j-1}$ and $1 \leq p \leq l_{j}^{q}$ and therefore $V \in \mathcal{A}_{n, m}\left(P_{j, i}\right)$ for all $P_{j, i} \subset Q_{j, p}^{q}$. Since there are $\left(k_{j}\right)^{m}$ such parallelepipeds $P_{j, i}$ and since $E \cap V \cap P_{j, i} \neq \emptyset$ for all of them, we need at least $\left(\frac{k_{j}}{3}\right)^{m} m$-cubes with side-length

$$
d_{j}=\frac{1}{k_{j}} \min _{\substack{1 \leq q \leq N_{j-1} \\ 1 \leq p \leq l_{j}^{q}}} e_{1}\left(Q_{j, p}^{q}\right)
$$

to cover $E \cap V$. Using the fact that

$$
k_{j} \geq\left(\min _{\substack{1 \leq q \leq N_{j-1} \\ 1 \leq p \leq l_{j}^{q}}} e_{1}\left(Q_{j, p}^{q}\right)\right)^{-j m+1}
$$

we have $\left(k_{j}\right)^{j m} \geq\left(d_{j}\right)^{1-j m}$ which gives $\operatorname{dim}_{\mathrm{B}}(E \cap V)=m$ where $\operatorname{dim}_{\mathrm{B}}$ is the upper box-counting dimension (for the definition see [F2, Chapter 3] or [Mat3, Chapter 5]). Similarly we see that $\operatorname{dim}_{\mathrm{B}}(E \cap V \cap O)=m$ for all open sets $O \subset \mathbb{R}^{n}$ with $E \cap V \cap O \neq \emptyset$, and so [F2, Corollary 3.9] gives $\operatorname{dim}_{\mathrm{p}}(E \cap V)=m$. This completes the proof since in Lemma 3.1 the set $B$ can be chosen in such a way that for all $V \in B$ the set $\left\{a \in V^{\perp}: V_{a} \in \mathcal{A}_{n, m}\left(P_{1,1}\right)\right\}$ is open.

\section{Bending Maps and Packing Dimensions of Sections}

In this section we shall indicate another difference between the behavior of Hausdorff and packing dimensions of sections of sets. By (1.3), (1.4), and the preservation of Hausdorff dimension under smooth mappings, the typical Hausdorff dimension of sections of a smooth image of a set is the same as the typical Hausdorff dimension of sections of the original set. We shall show that the packing dimensions of sections can change very radically under smooth diffeomorphisms. For simplicity, we shall do this only in the plane, although the techniques of the previous sections could certainly be used to prove similar results in higher dimensions.

Theorem 5.1. Let $f: A \rightarrow B$ be a $C^{2}$-diffeomorphism between open subsets $A$ and $B$ of $\mathbb{R}^{2}$. Suppose that $f$ does not map every line segment of $A$ onto a line segment. Then there is a compact subset $E$ of $A$ such that

1. $\mathcal{H}^{1}\left(\operatorname{proj}_{L^{\perp}}(E)\right)=0$ for $\gamma_{2,1}$-almost all $L \in G_{2,1}$, and

2. for all $L \in G_{2,1}$ we have $\operatorname{dim}_{\mathrm{p}}\left(f(E) \cap L_{a}\right)=1$ for all $a \in I_{L}$, where $I_{L}$ is some non-empty open subinterval of $L^{\perp}$. 
The proof is a slight modification of the methods of Section 4 and [Mat2] and therefore we shall only sketch it. We recall some terminology and notation from [Mat2]. From now on a parallelogram will always mean a non-degenerate closed parallelogram in $\mathbb{R}^{2}$ whose shorter sides are parallel to the $x_{1}$-axis. Given a $C^{1}$-curve $\mathcal{C}$ and a parallelogram $P$, we say that $\mathcal{C} \in \Gamma(P)$ if $\mathcal{C} \cap P$ has a connected component meeting both of the longer sides of $P$ but neither of the shorter ones. We denote by $\operatorname{dir}(\mathcal{C}, x)$ the direction of the tangent of $\mathcal{C}$ at $x \in \mathcal{C}$. Finally, $\mathrm{p}_{\theta}=\operatorname{proj}_{l_{\theta}^{\perp}}$ where $l_{\theta}=\{t(\cos \theta, \sin \theta): t \in \mathbb{R}\}$ for $\theta \in[0, \pi)$.

Lemma 5.2. Let $P$ be a parallelogram, $\varepsilon>0,0<s<1,0<\alpha<\frac{\pi}{10}$, and let $k_{\alpha} \geq 1$ be the largest integer with $5\left(k_{\alpha}+1\right) \alpha<\pi$. Then there is a finite family $\mathcal{P}$ of subparallelograms of $P$ with the following properties:

(1.) $\mathcal{H}^{1}\left(\mathrm{p}_{\theta}(\cup \mathcal{P})\right) \leq \varepsilon$ for $5 i \alpha \leq \theta \leq(5 i+1) \alpha, i=1, \ldots, k_{\alpha}$.

(2.) If $\mathcal{C} \in \Gamma(P)$ with $\operatorname{dir}(\mathcal{C}, x) \notin((5 i-1) \alpha,(5 i+2) \alpha)$ for all $i=1, \ldots, k_{\alpha}$, $x \in \mathcal{C}$, then there are parallelograms $P_{1}, \ldots, P_{l} \in \mathcal{P}$ having the same side-length $d$ for their shorter sides such that $l d^{s}>1$ and $\mathcal{C} \in \Gamma\left(P_{i}\right)$ for all $i=1, \ldots, l$.

Proof. [Mat2, Lemma 3] gives a finite family $\mathcal{R}$ of subparallelograms of $P$ for which (1) is satisfied and if $\mathcal{C}$ is as in (2), then $\mathcal{C} \in \Gamma(Q)$ for some $Q \in \mathcal{R}$. Subdividing each parallelogram of $\mathcal{R}$ into sufficiently many subparallelograms we get the required family $\mathcal{P}$.

We can now use the argument in [Mat2, pp. 307-309]. First we choose a small open subset $U$ of $A$ such that $f$ bends many line segments in $U$. We may not be able to get this for all line segments in $U$, but if we stay away from some exceptional directions as described in [Mat2, Lemma 1] we find a subinterval $I$ of $[0, \pi)$ of length $\frac{1}{2}$ such that for line segments $J$ whose direction is in $I, f(J)$ is not a line segment. Using Lemma 5.2 we construct a compact set $F$ with the following properties:

(5.1) $F=\bigcap_{m=1}^{\infty} \bigcup \mathcal{P}_{m}$ where $\left(\mathcal{P}_{m}\right)$ is a nested sequence of subparallelograms of $U$.

(5.2) $\mathcal{H}^{1}\left(\mathrm{p}_{\theta}(F)\right)=0$ for almost all $\theta \in[0, \pi)$.

(5.3) For all $\theta \in I$ we have $\operatorname{dim}_{\mathrm{p}}\left(f(F) \cap\left(l_{\theta}+a\right)\right)=1$ for all $a \in I_{\theta}$, where $I_{\theta} \subset \mathbb{R}$ is some non-empty open subinterval of $l_{\theta}^{\perp}$.

The set $F$ can be taken to be one of the sets $E_{n}$ in [Mat2, p. 307] (for example, $F=E_{6}$ if we take $\varepsilon=\frac{1}{6}$ in the application of [Mat2, Lemma 1] when 
choosing the set $U$ above). Then (5.1) and (5.2) are satisfied. To get (5.3) we choose a sequence $s_{m} \in(0,1)$ with $\lim _{m \rightarrow \infty} s_{m}=1$. Proceed otherwise exactly as in the last four lines of [Mat2, p. 307] and the first ten lines of [Mat2, p. 308] but instead of applying [Mat2, Lemma 3] apply Lemma 5.2 with $s=s_{m}$. This gives the family $\mathcal{P}_{m+1}$. As in the last paragraph in [Mat2, p. 308] we see that for all $\theta \in I$ we have $f(F) \cap\left(l_{\theta}+a\right) \neq \emptyset$ for $a \in I_{\theta}$ (which is an open subinterval of $l_{\theta}^{\perp}$ ). (There is a misprint in [Mat2, p. 308]: the first sentence of the last paragraph should read $\left(l_{\theta}+a\right) \cap f\left(E_{n}\right) \neq \emptyset$ instead of $\left(l_{\theta}+a\right) \cap f\left(E_{n}\right)=\emptyset$.) Because of the stronger formulation of Lemma 5.2 we now know more: for any open set $U^{\prime}$ with $f(F) \cap U^{\prime} \neq \emptyset$ and for sufficiently large $m$ we need at the $m$-th stage at least $l_{m}$ intervals of length $d_{m}$ with $l_{m}\left(d_{m}\right)^{s_{m}}>1$ to cover $f(F) \cap U^{\prime} \cap\left(l_{\theta}+a\right)$ for $\theta \in I$ and $a \in I_{\theta}$. Further, $\lim _{m \rightarrow \infty} d_{m}=0$, and therefore $\operatorname{dim}_{\mathrm{p}}\left(f(F) \cap\left(l_{\theta}+a\right)\right)=1$ for $\theta \in I$ and $a \in I_{\theta}$. Since $I$ has length $\frac{1}{2}$, we can take as $E$ the union of seven suitably rotated copies of $F$.

\section{Acknowledgements}

The authors acknowledge the support of the Isaac Newton Institute, Cambridge, where part of this work was carried out. MJ thanks the Academy of Finland (grant number 38955) and Vilho, Yrjö and Kalle Väisälä Fund for financial support.

\section{References}

[C] M. Csörnyei, On planar sets with prescribed packing dimensions of line sections, in Math. Proc. Cambridge Philos. Soc. to appear.

[D] R. O. Davies, On accessibility of plane sets and differentiation of functions of two real variables, Proc. Cambridge Philos. Soc., 48 (1952), 215-232.

[F1] K. J. Falconer, Sets with prescribed projections and Nikodym sets, Proc. London Math. Soc. (3), 53 (1986), 48-64.

[F2] K.J. Falconer, Fractal Geometry: Mathematical Foundations and Applications, John Wiley \& Sons, (1990).

[F3] K. J. Falconer, Sets with large intersections, J. London Math. Soc., 49 (1994), 267-280. 
[FH1] K. J. Falconer and J. D. Howroyd, Projection theorems for box and packing dimensions, Math. Proc. Cambridge Philos. Soc., 119 (1996), 287-295.

[FH2] K. J. Falconer and J. D. Howroyd, Packing dimensions of projections and dimension profiles, Math. Proc. Cambridge Philos. Soc., 121 (1997), 269-286.

[FJ] K. J. Falconer and M. Järvenpää, Packing dimensions of sections of sets, Math. Proc. Cambridge Philos. Soc., 125 (1999), 89-104.

[FM] K. J. Falconer and P. Mattila, The packing dimension of projections and sections of measures, Math. Proc. Cambridge Philos. Soc., 119 (1996), 695-713.

[JM] M. Järvenpää and P. Mattila, Hausdorff and packing dimensions and sections of measures, Mathematika, 45 (1998), 55-77.

[K] R. Kaufmann, On Hausdorff dimension of projections, Mathematika, 15 (1968), 153-155.

[Mar] J. M. Marstrand, Some fundamental geometrical properties of plane sets of fractional dimensions, Proc. London Math. Soc. (3), 4 (1954), 257-302.

[Mat1] P. Mattila, Hausdorff dimension, orthogonal projections and intersections with planes, Ann. Acad. Sci. Fenn. Ser. A I Math., 1 (1975), $227-244$.

[Mat2] P. Mattila, Smooth maps, null-sets for integralgeometric measure and analytic capacity, Ann. of Math., 123 (1986), 303-309.

[Mat3] P. Mattila, Geometry of Sets and Measures in Euclidean Spaces, Cambridge University Press (1995). 